Article

\title{
Synthesis, Stability, and Antidiabetic Activity Evaluation of (-)-Epigallocatechin Gallate (EGCG) Palmitate Derived from Natural Tea Polyphenols
}

\author{
Bingbing Liu ${ }^{1,2} \oplus$, Zhengzhong Kang ${ }^{1}$ and Weidong Yan ${ }^{1, *}$ \\ 1 Department of Chemistry, Zhejiang University, Hangzhou 310027, China; 11637060@zju.edu.cn (B.L.); \\ kangzhzh@zju.edu.cn (Z.K.) \\ 2 State Key Laboratory of Environmental and Biological Analysis, Hong Kong Baptist University, \\ Kowloon Tong, Kowloon, Hong Kong 999077, China \\ * Correspondence: yanweidong@zju.edu.cn; Tel.: +86-571-8795-1430; Fax: +86-571-8795-1895
}

check for

updates

Citation: Liu, B.; Kang, Z.; Yan, W. Synthesis, Stability, and Antidiabetic Activity Evaluation of

(-)-Epigallo-catechin Gallate (EGCG)

Palmitate Derived from Natural Tea Polyphenols. Molecules 2021, 26, 393. https://doi.org/10.3390/molecules 26020393

Academic Editor: F. Javier Luque Received: 6 September 2020 Accepted: 8 January 2021

Published: 13 January 2021

Publisher's Note: MDPI stays neutral with regard to jurisdictional clai$\mathrm{ms}$ in published maps and institutional affiliations

Copyright: (C) 2021 by the authors. Licensee MDPI, Basel, Switzerland. This article is an open access article distributed under the terms and conditions of the Creative Commons Attribution (CC BY) license (https:// creativecommons.org/licenses/by/ $4.0 /)$.

\begin{abstract}
This work describes a novel approach for the synthesis of (-)-epigallocatechin gallate (EGCG) palmitate by a chemical-synthesis method, where the elevated stability of the EGCG derivative is achieved. Various parameters affecting the acylation process, such as the base, solvent, as well as the molar ratio of palmitoyl chloride, have been studied to optimize the acylation procedure. The optimized reaction condition was set as follows: EGCG/palmitoyl chloride/sodium acetate was under a molar ratio of 1:2:2, with acetone as the solvent, and the reaction temperature was $40^{\circ} \mathrm{C}$. Under the optimized condition, the yield reached $90.6 \%$. The EGCG palmitate (PEGCG) was isolated and identified as $4^{\prime}$-O-palmitoyl EGCG. Moreover, the stability of PEGCG under different conditions was proved significantly superior to EGCG. Finally, PEGCG showed better inhibition towards $\alpha$-amylase and $\alpha$-glucosidase, which was 4.5 and 52 times of EGCG, respectively. Molecular docking simulations confirmed the in vitro assay results. This study set a novel and practical synthetic approach for the derivatization of EGCG, and suggest that PEGCG may act as an antidiabetic agent.
\end{abstract}

Keywords: EGCG palmitate; molecular modification; stability; antidiabetic activity; molecular docking

\section{Introduction}

(-)-Epigallocatechin gallate (EGCG), a compound with versatile bioactivities, is the most abundant tea polyphenol in green tea leaves [1]. EGCG has specific health-promoting activities, including antioxidative, antibacterial, anticancer, cardioprotective effect, and cholesterol-lowering effect [2-6]. It is the first botanical drug approved by the US Food and Drug Administration (FDA) as an anti-infective prescription for genital warts. Besides, EGCG is found to possess antidiabetic activity. Wolfram et al. found that EGCG beneficially modified glucose and lipid metabolism in H4IIE cells and markedly enhanced glucose tolerance in diabetic rodents [7]. Also, hepatic glucose production was repressed by EGCG through a PI3K-dependent manner [8]. Kamiyama et al. found that green tea supplementation increased insulin sensitivity and showed the antidiabetic effect on animal models of insulin resistance [9].

Oral administration of EGCG is found to be first absorbed in the intestine, which is a slightly alkaline condition [10]. However, EGCG is unstable in an alkaline system and is easily oxidized. For example, Sang et al. found that EGCG is unstable in sodium phosphate buffer (pH 7.4), where 90\% of EGCG is lost in only $3 \mathrm{~h}[11,12]$. Moreover, EGCG is highly hydrophilic, which means that it cannot easily pass through the lipid bilayer cell membranes by passive diffusion, and will be quickly inactivated or irreversibly oxidized, thus leading to the low availability $[12,13]$. Previous studies showed that only $0.01 \%$ of EGCG was absorbed in rats when given $56 \mathrm{mg}$ of EGCG orally, and $0.32 \%$ of EGCG was absorbed in humans after a single oral intake of $97 \mathrm{mg}$ [14]. 
Therefore, to increase the metabolic stability and bioavailability of EGCG, various efforts have been made on the structural modification of EGCG [15-17]. The EGCG derivatives showed enhanced bioavailability, and among these, EGCG-C16 esters, or EGCG palmitates, are found to have excellent bioactivities, such as antivirus [18,19], antitumor [20,21], antibacterial, and antifungal activities [22]. For instance, Matsumura et al. found that EGCG-C16 suppressed tumor growth in colorectal tumor-bearing mice compared to EGCG [20]. An EGCG palmitate was found to act as a topical antiviral agent for herpes simplex virus (HSV) [18]. EGCG palmitate is a novel lipophilic antioxidant that is considered to be generally recognized as safe (GRAS) by the US FDA in 2019. The enhanced bioavailability of the EGCG-acyl ester derivatives may be attributed to the slow release of EGCG from these derivatives in vivo [16].

Synthesis of EGCG palmitate can be attained by the chemical-synthesis or enzymatic method $[15,19,23]$. Although enzymatic catalysis is regioselective under some conditions, it has the shortcomings of being expensive, time-consuming, and limited in mild conditions due to the physical properties of the enzymes, while chemical synthesis is fast, cheap, and has higher yields; chemical synthesis includes homogeneous catalysis and heterogeneous catalysis. As for homogeneous catalysis, the advantages include that the catalytic efficiency is higher, and the catalyst usage is less compared to using the heterogeneous catalyst, while for heterogeneous catalysis, its merit is that the catalyst can be easily removed from the reaction system. Comparison of the reported chemical-synthesis or enzymatic method of EGCG palmitate with the method developed in this study is listed in Table 1. It can be clearly seen that the method developed in this study has the merit of high-yield.

Table 1. Comparison of chemical-synthesis or enzymatic method of EGCG palmitate.

\begin{tabular}{|c|c|c|c|c|c|c|}
\hline Method & Product & Catalyst & Solvent & Reaction Time & Yield & Ref. \\
\hline Enzymatic method & $\begin{array}{l}\text { Mixture of four EGCG-C16 } \\
\text { regioisomers }\end{array}$ & Lipase PL (Alcaligenes sp.) & N,N-Dimethylformamide & $8 \mathrm{~h}$ & $35-39 \%$ & [19] \\
\hline Chemical-synthesis & $\begin{array}{l}\text { Mixture of four EGCG-C16 } \\
\text { regioisomers }\end{array}$ & Triethylamine & Tetrahydrofuran & $24 \mathrm{~h}$ & $12-16 \%$ & [19] \\
\hline Chemical-synthesis & EGCG-4'-O-palmitate & Pyridine & Ethyl acetate & $3 \mathrm{~h}$ & - & [24] \\
\hline Chemical-synthesis & EGCG-C16 tetraester & Pyridine & Ethyl acetate & - & $57 \%$ & [15] \\
\hline This method & EGCG-4'-O-palmitate & Sodium acetate & Acetone & $6 \mathrm{~h}$ & $90.6 \%$ & - \\
\hline
\end{tabular}

As for the esterification reaction, the kinds of catalysts can be divided into acid and base. The esterification of EGCG usually uses acyl chloride or anhydride as the acylation reagent, and bases like pyridine to act as the acid-binding agent that can react with the hydrogen chloride released in the esterification process. However, bases like pyridine are toxic $[15,24]$. Based on these facts, we decided to select a less toxic base for the esterification of EGCG. Some solid bases like sodium carbonate have been used in the esterification process [25], therefore, we listed some potential solid bases that might be applicable for this EGCG reaction. The toxicity of these solid bases was obtained from the Material Safety Data Sheet (MSDS) database and listed in Table 2. In addition, since Mori indicated that the positions of the acyl groups of EGCG derivatives do not affect their antiviral activities [19], we determined to develop a facile chemical-synthesis method to obtain the EGCG palmitate.

In this work, the objectives were to better understand the relationships between various reaction variables (i.e., usage of the reagent) and the responses (EGCG conversion, product yield), and to obtain the optimum synthesis condition for PEGCG. Also, the stability of the PEGCG under several conditions was studied and compared with EGCG. Finally, since many studies have reported the antidiabetic activity of EGCG and some EGCG ester derivatives $[8,9,26]$, we deduced that PEGCG may have the same or even higher activity. The antidiabetic activity of PEGCG was evaluated and compared with EGCG as well as standard drug acarbose by using in vitro assays. 
Table 2. Toxicity of some reagents and their harm to human health.

\begin{tabular}{|c|c|c|}
\hline Reagent & Acute Toxicity Test (Mouse) ${ }^{a}$ & Harm to Human Health a \\
\hline Sodium acetate & LD50 25,956 mg/kg, non-toxic & None \\
\hline Sodium carbonate & LD50 $4090 \mathrm{mg} / \mathrm{kg}$, mild toxicity & Irritant and corrosive \\
\hline Sodium bicarbonate & LD50 $4220 \mathrm{mg} / \mathrm{kg}$, mild toxicity & Slight irritation to the eyes \\
\hline Triethylamine & LD50 $460 \mathrm{mg} / \mathrm{kg}$, medium toxicity & $\begin{array}{l}\text { strong irritant to the respiratory tract; after inhaling can } \\
\text { cause pulmonary edema or even death }\end{array}$ \\
\hline Pyridine & LD50 1580 mg/kg, mild toxicity & Strong irritant; anesthetizes the central nervous system \\
\hline 4-dimethylaminopyridine (DMAP) & LD50 $250 \mathrm{mg} / \mathrm{kg}$, medium toxicity & Irritating to the eyes; eating poisonous \\
\hline
\end{tabular}

${ }^{a}$ The Acute toxicity and harm to human health were obtained from Material Safety Data Sheet (MSDS).

\section{Results and Discussion}

\subsection{Screen of Different Reaction Conditions}

A single-factor experiment was conducted to investigate the variables (i.e., the molar ratio of the palmitoyl chloride, base, and solvent) and the responses (conversion, product yield) of the reaction, in order to set the optimum reaction condition for the synthesis of PEGCG.

\subsubsection{Effect of the Molar Ratio of Palmitoyl Chloride}

To investigate the effect of the molar ratio of palmitoyl chloride on the reaction, the reaction temperature $\left(40^{\circ} \mathrm{C}\right)$, reaction time $(6 \mathrm{~h})$, the quantity of EGCG $(10 \mathrm{mM})$, and sodium acetate $(40 \mathrm{mM})$ were held constant, and only usages of the palmitoyl chloride were changed.

As shown in Figure 1a, the retention time (RT) of EGCG was $17.1 \mathrm{~min}$, and RT of PEGCG was $44.5 \mathrm{~min}$. As shown in Figure 1d, when the molar ratio of EGCG to palmitoyl chloride was 1:1, the conversion of EGCG was $94.3 \%$. When the molar ratio was 1:2, the EGCG conversion was enhanced to $99.3 \%$; when the molar ratio increased to $1: 3$ and 1:4, no EGCG peaks were observed in HPLC, and it was considered that the EGCG conversion reached nearly $100 \%$. However, even when the palmitoyl chloride was in excess, the product was still EGCG monopalmitate (Supporting Information: Figures S3 and S5). No esters of higher degrees (di-, tri-, or tetraesters) were formed, which might be ascribed to the steric hindrance of the long fatty acid chain that caused difficulty to the acylation process [15]. In addition, it may also be because the activity of each hydroxyl of the EGCG molecule is different. As reported before, hydroxyls on the B ring and D ring of EGCG are more reactive than other hydroxyls.

\subsubsection{Effect of Base}

Two molar equivalents of different solid bases (sodium acetate, sodium carbonate, and sodium bicarbonate) were used, and triethylamine was used as a conventional organic base to be compared with the three selected bases. The usage of palmitoyl chloride ( 2 molar equivalents) and other factors were held constant.

HPLC chromatograms of the mixtures obtained with the above are shown in Figure $1 b$. As shown in Table 3, EGCG conversions were nearly $100 \%$. Sodium acetate and triethylamine gave a high yield of PEGCG, which was $90.6 \%$ and $88.1 \%$, respectively, while it was low with sodium acetate $(57.1 \%)$ and sodium carbonate $(72.4 \%)$. Therefore, sodium acetate was considered as a suitable base and was used in the following experiments.

Table 3. Effect of the base on the acylation reaction.

\begin{tabular}{ccc}
\hline Base & EGCG Conversion/\% & Yield/\% ${ }^{\text {a }}$ \\
\hline Sodium acetate & nearly 100 & $90.6 \pm 2$ \\
Sodium carbonate & nearly 100 & $57.1 \pm 2$ \\
Sodium bicarbonate & nearly 100 & $72.4 \pm 2$ \\
Triethylamine & nearly 100 & $88.1 \pm 2$ \\
\hline a The results are calculated as mean + standard deviation of three replicates.
\end{tabular}

a The results are calculated as mean \pm standard deviation of three replicates. 

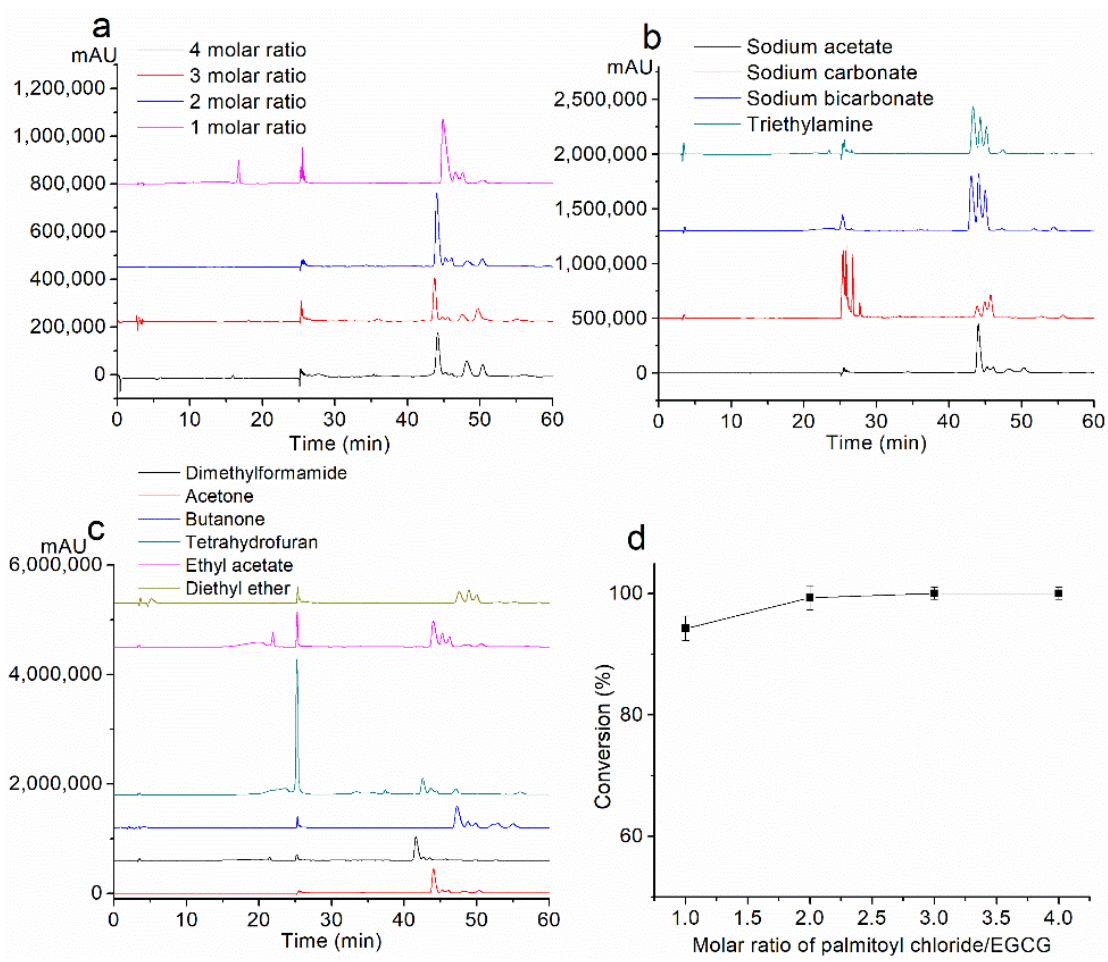

Figure 1. Effect of (a) molar ratio of palmitoyl chloride, (b) base, and (c) solvent, on the effect of the reaction by HPLC analysis. (d) EGCG conversion under the condition of 1-4 molar ratios of palmitoyl chloride.

\subsubsection{Effect of Solvent}

The 1-octanol/water partition coefficient, $\log \mathrm{P}$, is recognized as one of the principal parameters to evaluate the lipophilicity of chemical compounds. The compound of higher lipophilicity has a higher $\log P$ value and is considered relatively less polar. The lipophilicity of the EGCG, 4'-O-palmitoyl EGCG and different solvents were computationally obtained by ALOGPS 2.1 (http:/ /www.vcclab.org/lab/alogps/) [27].

The HPLC chromatograms of the products of different solvents are shown in Figure 1c. Under the conditions of 2 molar ratio of palmitoyl chloride and sodium acetate as the base, EGCG conversions were almost the same by using different solvents, and the products were mono-esters. However, as shown in Table 4, the yield of the product was not proportional to the polarity of the solvent; acetone showed the highest yield $(90.6 \%)$ and other solvents showed relatively lower yields (no more than $90.6 \%$ ). Dimethylformamide is a relatively polar solvent, while ethyl acetate and diethyl ether are relatively non-polar solvents. Since the $\log$ P value of the material (EGCG) is 2.38 and the product (PEGCG) is 6.48 , it might be speculated that acetone acts as a medium-polarity solvent that offers a compromised dissolution condition between the materials and the product. It had been found that 10 mmol EGCG was partly dissolved in ethyl acetate and diethyl ether, respectively, which might explain the low yield with the corresponding solvent.

Table 4. Effect of solvent on the acylation reaction.

\begin{tabular}{cccc}
\hline Solvent & Log $\mathbf{P}$ & EGCG Conversion $/ \mathbf{\%}^{\mathbf{a}}$ & Yield $/ \mathbf{\%}^{\mathbf{a}}$ \\
\hline Dimethylformamide & -0.77 & $95.6 \pm 2$ & $45.3 \pm 3$ \\
Acetone & -0.29 & nearly 100 & $90.6 \pm 2$ \\
Butanone & 0.41 & nearly 100 & $66.2 \pm 3$ \\
Tetrahydrofuran & 0.35 & $92.5 \pm 1$ & $39.4 \pm 3$ \\
Ethyl acetate & 0.74 & $92.3 \pm 1$ & $79.3 \pm 2$ \\
Diethyl ether & 1.12 & nearly 100 & $47.6 \pm 2$ \\
\hline
\end{tabular}

a The results were calculated as mean \pm standard deviation of three replicates. 
Based on these results, acetone was considered the most desirable solvent.

In conclusion, considering the reaction variables on the effect of the reaction, the optimum reaction condition was set as follows: sodium acetate as the base, acetone as the solvent, and the molar ratio of palmitoyl chloride was 2 . Under the optimum reaction condition, the yield of EGCG palmitate could reach $90.6 \%$.

\subsection{Stability under the Alkalescent Condition}

According to previous studies, EGCG was extremely vulnerable to degrading in alkaline solutions. If EGCG were not well absorbed, part of the mechanism might include the selective degradation of EGCG in the intestine where the $\mathrm{pH}$ is neutral or alkaline. The oxidation products of EGCG are two EGCG dimers: P2 (Mw 884) and Theasinensin (Mw 914) [11,28].

Firstly, the oxidation products of EGCG in ethanol/PBS solution were identified as P2 (RT $23.199 \mathrm{~min}$ ) and Theasinensin (RT $23.199 \mathrm{~min}$ ) by HPLC-MS (Figure S6).

Then, the stability of EGCG and PEGCG under the alkalescent condition was compared. As shown in Figure 2a,b, under the alkaline condition in the open air, EGCG immediately turned into its dimers once added into the solution. After $1 \mathrm{~h}, 93 \%$ of the EGCG was degraded. After $5 \mathrm{~h}$, the EGCG was utterly degraded. While for PEGCG, most of the PEGCG was hydrolyzed to EGCG and its dimers after $1 \mathrm{~h}$. After $5 \mathrm{~h}, 100 \%$ of the PEGCG was hydrolyzed to EGCG and then oxidized to EGCG dimers. The above results indicate that the PEGCG is significantly more stable than EGCG under the alkalescent condition.
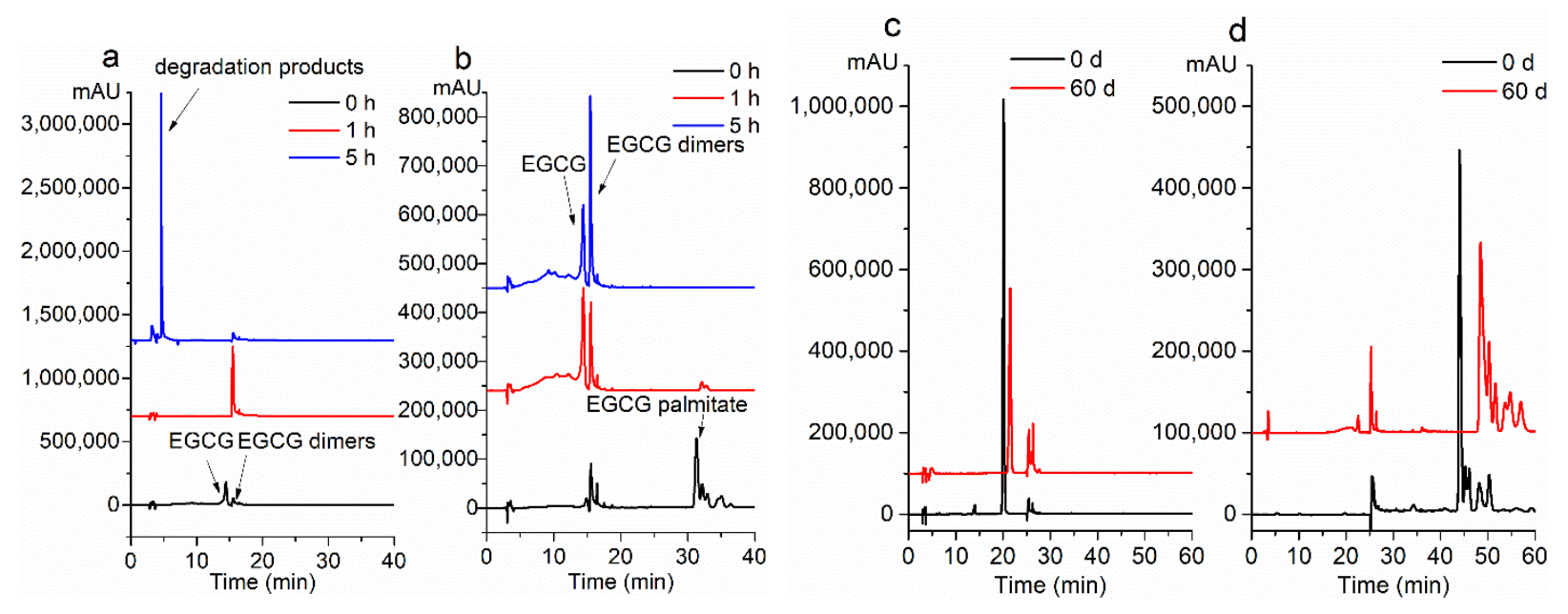

Figure 2. Stability evaluation under the alkalescent condition of (a) EGCG, and (b) PEGCG by HPLC-MS analysis. Storage stability evaluation of (c) EGCG, and (d) PEGCG by HPLC-MS analysis.

\subsection{Storage Stability}

After 60 days in the open air, the white-colored EGCG powder turned to reddishbrown upon long-term storage, while the color of the PEGCG remained nearly the same. It showed that $78 \%$ of EGCG palmitate remained after being stored for $60 \mathrm{~d}$ at room temperature (Figure 2c), while EGCG was $45 \%$ remained (Figure 2d). This indicates that PEGCG keeps better stability in the air, making it is more convenient for storage.

\subsection{Thermal Stability}

As shown in Figure S7, the thermal decomposition temperature of EGCG was $234.5^{\circ} \mathrm{C}$, while the thermal decomposition temperature of PEGCG was $311.19^{\circ} \mathrm{C}$. PEGCG had better thermal stability compared with EGCG, which is vital since PEGCG can be used as an antioxidant in edible oil.

Edible oil is usually used at a temperature under $250{ }^{\circ} \mathrm{C}$. At this time, PEGCG, a lipid-soluble antioxidant, can still keep stable and play the antioxidant role at high 
temperatures, protecting the nutritional components of edible oil from being destroyed. What is more, acrylamide and pyridine heterocyclic amine (PHIP), the carcinogenic and mutagenic compounds, are quickly produced when foods are frying at high temperatures. The inhibitory effects of polyphenols on acrylamide and PHIP have been reported [29,30]. Given the excellent thermal stability and antioxidant property of PEGCG palmitate, it is speculated that PEGCG could also inhibit the production of acrylamide and PHIP, which deserves further study.

\subsection{Inhibition of $\alpha$-Amylase}

According to various in vivo studies, inhibition of $\alpha$-amylase and $\alpha$-glucosidase is assumed to be one of the most effective approaches for diabetes care [31]. However, several commercial antidiabetic drugs, such as acarbose, miglitol, voglibose, and sitagliptin, have positive effects on glycemic values after food intake, but their lack of specificity has produced some gastrointestinal side effects, like abdominal cramping, flatulence, and diarrhea [32,33]. Natural $\alpha$-glucosidase and $\alpha$-amylase inhibitors are therefore being investigated as new candidates to control hyperglycemia in diabetic patients, for they do not cause severe side effects and may also be beneficial in weight reduction for people consuming large amounts of starch [34].

For $\alpha$-amylase inhibition, as shown in Table 5, the inhibitory activity in descending order was acarbose $\left(\mathrm{IC}_{50} 1.10 \mu \mathrm{M}\right)>$ PEGCG $\left(\mathrm{IC}_{50} 1.64 \mu \mathrm{M}\right)>\mathrm{EGCG}\left(\mathrm{IC}_{50} 7.44 \mu \mathrm{M}\right)$ (Figure 3). It is notable that the inhibitory activity of PEGCG was 4.5 times higher than EGCG, and was comparable to acarbose. The elevated inhibitory activity of PEGCG may be attributed to its increased stability and bioavailability.

Table 5. Inhibition of $\alpha$-amylase and $\alpha$-glucosidase.

\begin{tabular}{ccc}
\hline Compound & IC $_{\mathbf{5 0}}(\boldsymbol{\mu} \mathbf{M})$ for $\boldsymbol{\alpha}$-Amylase & IC $_{\mathbf{5 0}}(\boldsymbol{\mu M})$ for $\boldsymbol{\alpha}$-Glucosidase \\
\hline EGCG & $7.44 \pm 0.05$ & $11.50 \pm 0.02$ \\
PEGCG & $1.64 \pm 0.04$ & $0.22 \pm 0.03$ \\
Acarbose & $1.10 \pm 0.03$ & $0.15 \pm 0.02$ \\
\hline
\end{tabular}

\subsection{Inhibition of $\alpha$-Glucosidase}

Similarly, the inhibition of PEGCG on $\alpha$-glucosidase was significantly improved compared to EGCG. It was found that $\mathrm{IC}_{50}$ values of EGCG, PEGCG, and acarbose were $11.50,0.22$, and $0.15 \mu \mathrm{M}$, respectively. Thus, the $\alpha$-glucosidase inhibitory activity of PEGCG was 52 times higher than EGCG and 0.68 times of acarbose. This result indicated that the PEGCG strongly suppressed the $\alpha$-glucosidase activity and has the possibility of controlling postprandial hyperglycemia.

The catalytic sites of amylase are ASP231, GLU261, and ASP328, which are located at the bottom of the active pocket. As shown in Figure 4, drug molecules are all in the pocket and binding directly to the catalytic sites. It indicates that the possible inhibition mechanism is achieved by occupying the catalytic sites to prevent the binding of substrates (sugar). It can be seen from Figure 4 that the binding conformations of the three drugs are different. Acarbose covered more catalytic sites than the other two drugs, which may be the reason why acarbose had better inhibitory effect. The three aromatic rings of EGCG could not completely cover the catalytic sites due to the spatial structure. More interestingly, the tail chain of PEGCG could not only anchor on the enzyme to assist binding but also bind to the catalytic sites (as shown in Figure 4, binding model 1). This may be the reason why PEGCG has a better inhibition effect than EGCG. 

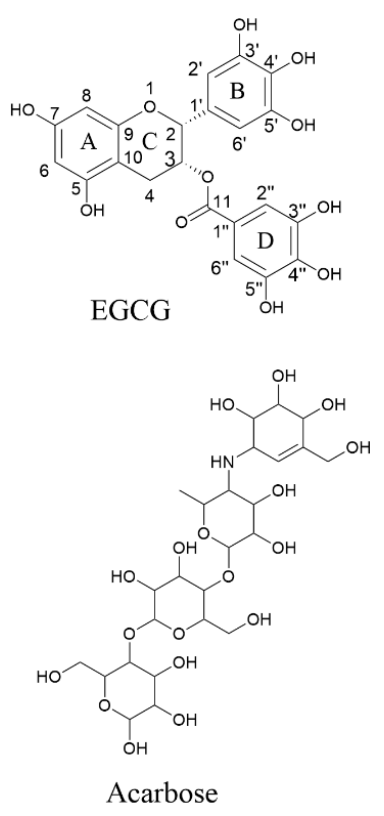

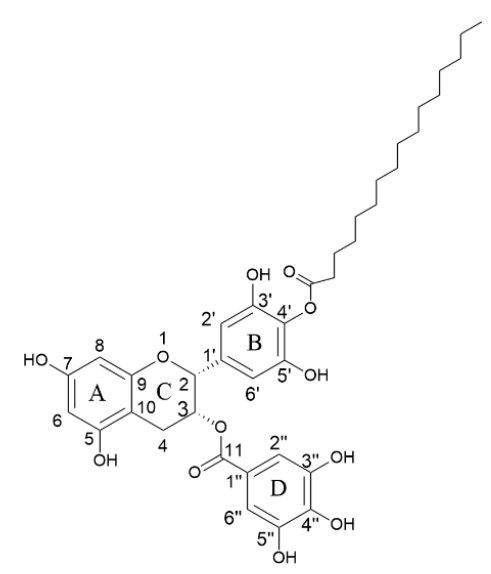

PEGCG

Figure 3. Chemical structure of the ligands from PubChem EGCG, PEGCG, and acarbose.

\subsection{Molecular Docking of the Ligands with $\alpha$-Amylase}

Here we used the molecular docking method to obtain the possible binding conformation of drugs and enzyme. Ten most likely binding structures are produced from AutoDock software. We compare the binding energy, contact atom number, and contact surface of the ten conformations (Figures S9 and S10). The top 3 binding conformations are listed in Figures 4 and 5, while other possible binding structures are shown in Figures S11-S14.

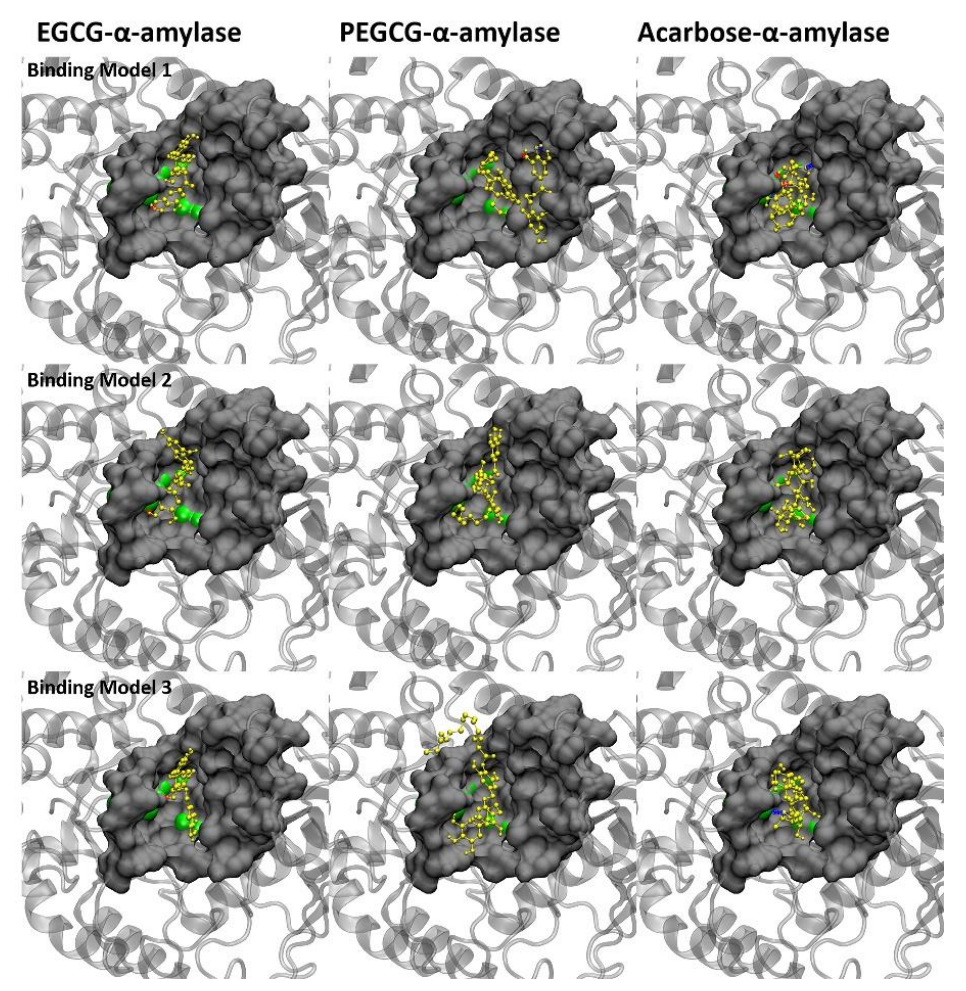

Figure 4. Top 3 predicted binding models of the ligands with $\alpha$-amylase. Protein is shown in silver in the cartoon model. Drugs are shown in yellow. The catalytic sites are displayed in green. The ligand molecules are represented in yellow. Amino acids in contact are displayed in the surface model. The red and blue dotted lines stand for hydrogen bonds. 


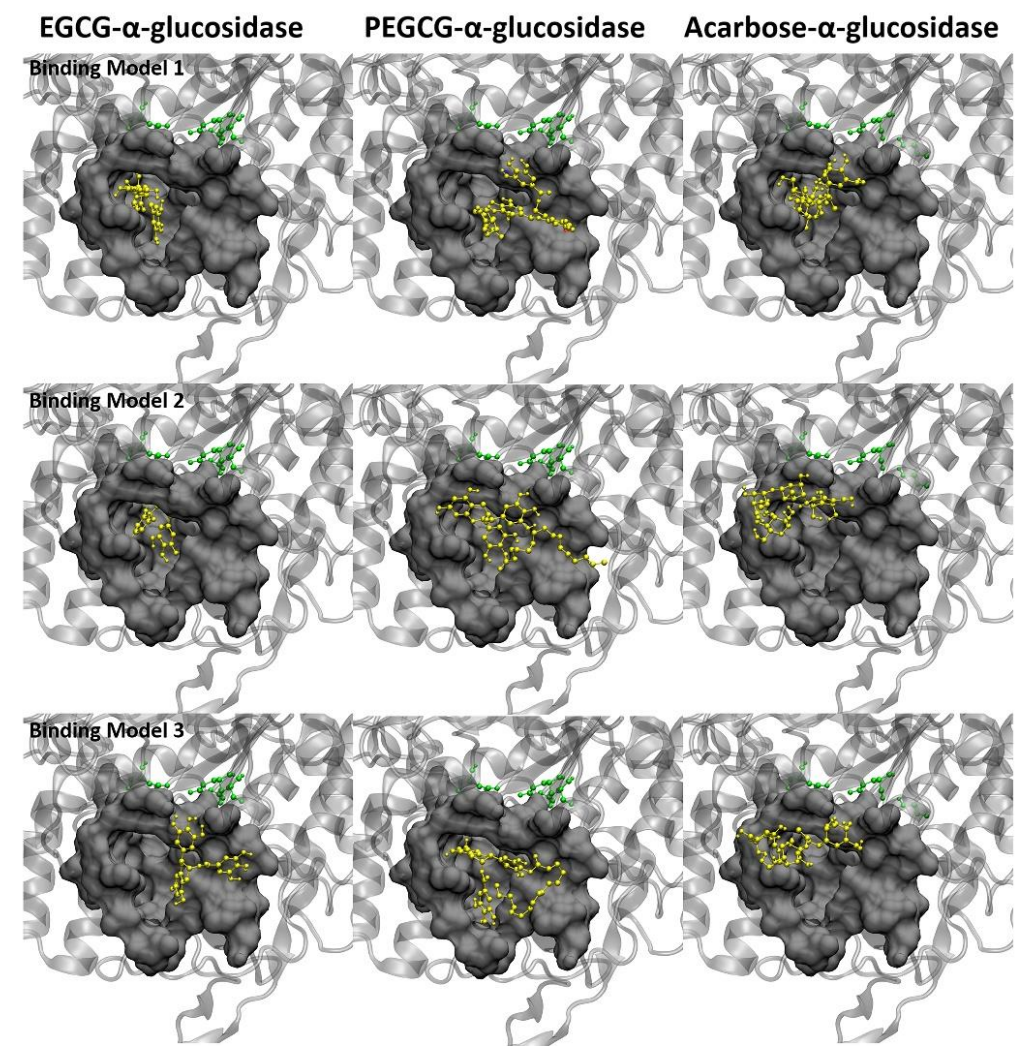

Figure 5. Top 3 predicted binding models of the ligands with $\alpha$-glucosidase. Protein is shown in silver in the cartoon model. Drugs are shown in yellow. The catalytic sites are displayed in green. The ligand molecules are represented in yellow. Amino acids in contact are displayed in the surface model. The red and blue dotted lines stand for hydrogen bonds.

Due to the limitation of AutoDock software, we could not get the accurate binding free energy of ligand-enzyme. We simply used the number of amino acids in contact and hydrogen bonds as the representative of van der Waals interaction and electrostatic interaction, respectively. The number of contact residues and number of the hydrogen bonds are listed in Table 6 (Binding details are shown in Table S1). As shown in Table 7, acarbose has the maximum number of hydrogen bond donor and acceptor counts. For PEGCG, the palmitoyl structure does not increase the number of hydrogen bond donors and acceptors.

Table 6. Binding overview of the ligands with $\alpha$-amylase.

\begin{tabular}{cccc}
\hline$\alpha$-Amylase & EGCG & PEGCG & Acarbose \\
\hline Number of contact residues & 20 & 22 & 19 \\
Number of the hydrogen bonds & 4 & 2 & 4 \\
\hline
\end{tabular}

Table 7. Hydrogen bond donor and acceptor count of the ligand.

\begin{tabular}{ccc}
\hline Inhibitor & Hydrogen Bond Donor Count & Hydrogen Bond Acceptor Count \\
\hline EGCG & 8 & 11 \\
PEGCG & 7 & 12 \\
Acarbose & 14 & 19 \\
\hline
\end{tabular}




\subsection{Molecular Docking of the Ligands with $\alpha$-Glucosidase}

Different from amylase, the catalytic sites ASP215, GLU277, and ASP352 of glucosidase are located below the pocket, and the substrate needs to pass through the pocket before binding to the catalytic site.

The three ligand molecules are all inserted into the catalytic pocket in Figure 5, without direct contact with the catalytic site. Therefore, the possible inhibition of the ligand on glucosidase is achieved by blocking the pocket that leads to the catalytic site. It can be seen from the structure figure that the aromatic structures of EGCG and acarbose are deeply inserted into the pocket and stay close to the catalytic sites, which successfully blocks the possible binding of substrate. As for PEGCG, both aromatic structure and palmitoyl structure can enter the pocket. As shown in binding model 1 (Figure 5), the palmitoyl part with suitable length can be folded into the pocket or anchor on the edge of the pocket to assist binding. At the same time, the hydrogen bond number is displayed as EGCG (3) $<$ PEGCG (4) < acarbose (5), and the number of amino acids in contact is EGCG (19) < acarbose (26) < PEGCG (27) in Table 8 (Binding details are shown in Table S2). Interestingly, the PEGCG is much closer to acarbose in terms of amino acid numbers in contact and hydron bond numbers.

Table 8. Binding overview of the ligands with $\alpha$-glucosidase.

\begin{tabular}{cccc}
\hline$\alpha$-Glucosidase & EGCG & PEGCG & Acarbose \\
\hline Number of contact residues & 19 & 27 & 26 \\
Number of the hydrogen bonds & 3 & 4 & 5 \\
\hline
\end{tabular}

We also observed that the number of contact atoms and the contact area of PEGCG were also close to that of acarbose and larger than EGCG (Figure S10). This indicates that although the mother nuclei structures of PEGCG and acarbose are different, they show similar characteristics in binding with glucosidase. This similarity was also found in the IC50 data of PEGCG and acarbose. From the binding conformations in Figure 5, we found that in addition to blocking the binding pocket, some part of PEGCG and acarbose are anchored at the edge of the pocket, while EGCG could only stay inside the pocket. Combined with IC50 data, we speculate that glucosidase may prefer inhibitors with a long chain, which not only block the inside of the pocket, but also cover the opening of the pocket.

\section{Experimental Section}

\subsection{Materials}

EGCG (from green tea, purity 92\%) was supplied by Pulimeidi Inc. (Hangzhou, China). Palmitoyl chloride, $\alpha$-amylase (from rhizobacteria Bacillus Licheniformis, EC 3.2.1.1), and acetonitrile of HPLC grade were purchased from Sigma-Aldrich Co. (St. Louis, MO, USA). $\alpha$-Glucosidase (from yeast Saccharomyces Cerevisiae, EC 3.2.1.20, 18.5 unit/mg) was purchased from Sinopharm Group Chemical Reagent Co. (Shanghai, China). Sodium acetate, sodium carbonate, sodium bicarbonate, and triethylamine were from Sinopharm Group Chemical Reagent Co., Ltd. (Shanghai, China). All of the other solvents and chemicals used were obtained from Sinopharm Group Chemical Reagent Co., Ltd. (Shanghai, China) and were of analytical grade. Sodium phosphate buffer (PBS) was purchased from Solarbio Co. (Beijing, China) and was prepared to make a concentration of $0.01 \mathrm{M}$ aqueous solution ( $\mathrm{pH}$ 7.2).

\subsection{Synthesis Procedure}

In a typical one-step synthesis protocol, $10 \mathrm{mmol}$ EGCG $(4.98 \mathrm{~g})$ was added to $100 \mathrm{~mL}$ of a solvent in a $250 \mathrm{~mL}$ flask immersed in a water bath $\left(40^{\circ} \mathrm{C}\right)$. After complete dissolution, the base was added to the solution. Subsequently, different molar ratios of palmitoyl chloride (1, 2, 3, or 4 molar ratio) were dropwise added to the solution, respectively, with 
mechanical stirring (100 rpm). After $6 \mathrm{~h}$, the reaction mixture was filtered and washed with $100 \mathrm{~mL}$ deionized water. Then $100 \mathrm{~mL}$ ethyl acetate was added for the extraction of the acylation product. And the organic phase was washed 2 times with deionized water. After that, the upper organic phase was dried with anhydrous sodium sulfate, concentrated under reduced pressure, and lyophilized. The lyophilized products were preserved at $-20{ }^{\circ} \mathrm{C}$ for further use.

\subsection{HPLC-MS Analysis}

The composition of the acylation mixture under the optimum reaction condition was determined by HPLC-MS, which used an Agilent 1290 HPLC unit (Agilent Technologies, Palo Alto, CA, USA) with a UV detector and a Cosmosil ODS C18 column $(4.6 \mathrm{~mm} \times$ $250 \mathrm{~mm}, 5 \mu \mathrm{m}$; Nacilai Tesque Inc., Kyoto, Japan). Eluent A and eluent B were acetonitrile $/$ water $=10: 90(v / v)$, and acetonitrile/water $=80: 20(v / v)$, each containing $0.2 \%$ formic acid $(v / v)$. A gradient elution program was as follows: 0-20 min, linear gradient $0-10 \% \mathrm{~B}$; 21-60 $\mathrm{min}, 88 \%$ B isocratic. The flow rate was $1.0 \mathrm{~mL} / \mathrm{min}$, and fractions were detected at $275 \mathrm{~nm}$. Mass spectroscopic (MS) analysis was performed using a 6460 Triple Quad MS detector system (Agilent Technologies, Palo Alto, CA, USA). The eluent was introduced into an electrospray source at negative mode (desolvation temperature $325^{\circ} \mathrm{C}$, capillary voltage $3.5 \mathrm{kV}$, nebulizer $45 \mathrm{psi}$ ). Argon was used as the collision gas (collision energy $16 \mathrm{eV}$ ), and nitrogen (dry gas flow, $5 \mathrm{~L} / \mathrm{min}$ ) was the desolvation gas.

\subsection{Purification and Identification of the Acylation Product}

Flash column chromatography was used to separate individual EGCG palmitate. $10 \mathrm{~g}$ of EGCG derivatives were eluted on a silica column with a mixture of petroleum ether/ethyl acetate/acetic acid $(3: 1: 0.05, v / v / v)$. The fractions were monitored by TLC (petroleum ether/ethyl acetate/acetic acid $=1: 1: 0.05, v / v / v$ ), and the predominant fraction was collected and washed three times with deionized water. Solvents were removed using a rotary evaporator. The predominant fraction (compound 1) was analyzed by NMR for its specific structure. The melting point of compound 1 was obtained by the differential scanning calorimetry (DSC) analysis on a DSC instrument (Q100, TA Instruments, New Castle, DE, USA).

The crude products were separated into different fractions by silica column chromatography, and the predominant one was collected and identified by NMR, which was reported in our previous study [35].

Theoretically, the molecular weight of EGCG monopalmitate is 696.8, and EGCG is 458.4 Da. As shown in Figure 6b, the selective ion of $\mathrm{m} / \mathrm{z} 695$ indicated that peaks 2, 3, and 4 might be EGCG monopalmitate. However, only the MS spectrum of peak 2 showed $\mathrm{m} / \mathrm{z} 695.3\left([\mathrm{M}-\mathrm{H}]^{-}\right)$and $\mathrm{m} / \mathrm{z} 238$ ([M-H-palmitoyl $]^{-}$), which is the indication of EGCG monopalmitate (PEGCG). Peak 3 and 4 were identified as the byproducts (Figure $6 \mathrm{~d}$ and Figure S3e). According to Figure S3g,h, mass spectra of peak 5 and 6 showed molecular ion peak of $\mathrm{m} / \mathrm{z} 737$, indicating that the components were not EGCG monopalmitate, either.

The ${ }^{1} \mathrm{H}$ and ${ }^{13} \mathrm{C}$ NMR analyses of PEGCG were as follows (Figures in Figures S1 and S2): PEGCG: yield 90.6\%; white crystal; m.p. $96.7^{\circ} \mathrm{C}$ (Figure S8); ${ }^{1} \mathrm{H}$ NMR (400 MHz, DMSO- $d_{6}$ ). $\delta 9.17$ (br, OH), 6.81 (s, 2H, H-2" , H-6") 6.41 (s, 2H, H-2' $\left.{ }^{\prime} \mathrm{H}^{\prime} 6^{\prime}\right), 5.93$ (d, J = 2.2 Hz, 1H, H-8), $5.82(\mathrm{~d}, \mathrm{~J}=2.2 \mathrm{~Hz}, 1 \mathrm{H}, \mathrm{H}-6), 5.33$ (s, 1H, H-3), 4.94 (s, 1H, H-2), 2.90 (m, 1H, H-4a), 2.65 (m, $J=16.0 \mathrm{~Hz}, 1 \mathrm{H}, \mathrm{H}-4 \mathrm{~b}), 2.27$ (t, $J=7.4 \mathrm{~Hz}, 2 \mathrm{H}, \mathrm{H}-\mathrm{p}-2), 1.50$ (m, 2H, H-p-2), 1.09-1.23 (m, $J=53.6 \mathrm{~Hz}, 24 \mathrm{H}, \mathrm{H}-\mathrm{p}-4$ to 15), 0.85 (t, $J=6.8 \mathrm{~Hz}, 3 \mathrm{H}, \mathrm{H}-\mathrm{p}-16), 4.09$ (br, OHs).

${ }^{13} \mathrm{C}$ NMR (101 MHz, DMSO-d 6 ). $\delta 173.44$ (C-p-1), 165.31 (C-11), 156.58 (C-7), 156.53 (C-9), 155.66 (C-5), 145.69 (C-3", C-5") $145.48\left(\mathrm{C}-3^{\prime}, \mathrm{C}^{\prime \prime} 5^{\prime}\right), 138.83\left(\mathrm{C}-4^{\prime \prime}\right), 132.40\left(\mathrm{C}-4^{\prime}\right), 128.65$ $\left(\mathrm{C}-1^{\prime}\right), 119.10\left(\mathrm{C}-1^{\prime \prime}\right), 108.67\left(\mathrm{C}-2^{\prime \prime}, \mathrm{C}-6^{\prime \prime}\right), 105.51\left(\mathrm{C}-2^{\prime}, \mathrm{C}-6^{\prime}\right), 99.59$ (C-10), 97.38 (C-8), 94.35 (C-6), 76.54 (C-2), 68.08 (C-3), 33.31 (C-p-2), 31.36 (C-p-3), 28.50-29.09 (C-p-4 to 13), 25.76 (C-4), 24.48 (C-p-4), 22.16 (C-p-15), 14.01 (C-p-16). P, palmitoyl. 

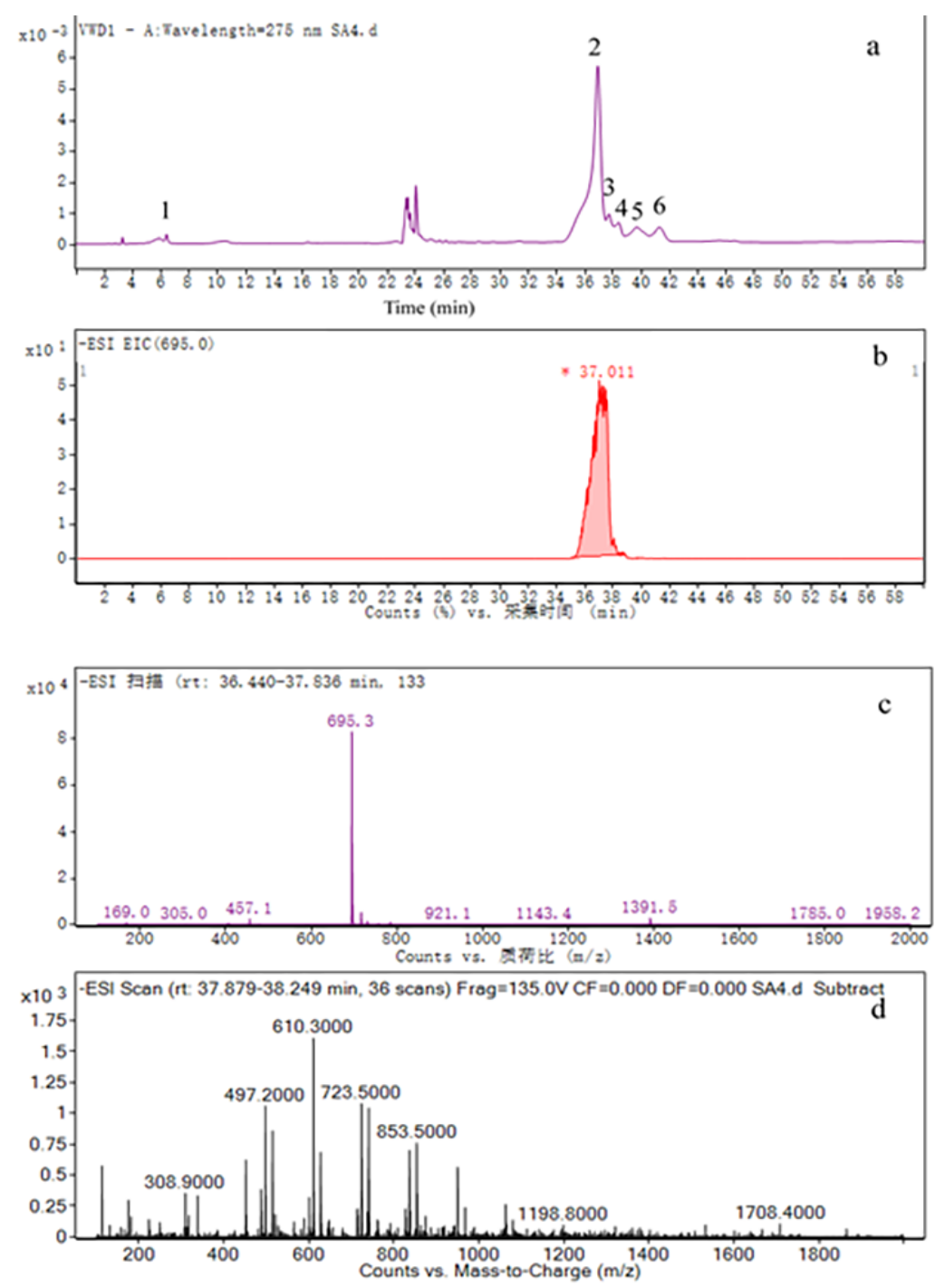

Figure 6. HPLC-MS analysis of product under the optimum condition (EGCG/palmitoyl chloride/ sodium acetate $=1: 2: 2$ ), (a) chromatogram of the product; (b) selective ion chromatogram of PEGCG (m/z 695); (c) mass spectra of PEGCG (Mw 696.8); (d) mass spectra of peak 3.

Based on the HPLC-MS and NMR analyses, PEGCG was identified as 4'-O-palmitoyl EGCG (Figure 3, molecular structures coincide with PubChem), where acylation occurs on the B ring of the EGCG molecule. As reported by Chen et al. [24], the same 4'-O-palmitoyl EGCG was synthesized and identified. It has been reported that hydroxyls on the $B$ ring of EGCG are more reactive [36-38], where the oxidation and acylation first occur.

\subsection{HPLC Analysis of the Acylation Reaction Process}

To further analyze the mixtures of different reaction conditions (base, solvent, molar ratio of palmitoyl chloride), the lyophilized crude products were dissolved with acetonitrile and filtered respectively. $20 \mu \mathrm{L}$ of the above solution was subjected to HPLC analysis (Wufeng LC-100 HPLC-UV, Shanghai, China) and separated on a Cosmosil ODS C18 column (4.6 mm $\times 250 \mathrm{~mm}, 5 \mu \mathrm{m}$; Nacilai Tesque Inc., Kyoto, Japan). The detection wavelength was set at $275 \mathrm{~nm}$, and the column temperature was $30^{\circ} \mathrm{C}$. The flow rate was $1.0 \mathrm{~mL} / \mathrm{min}$. The elution program was the same as HPLC-MS as described above. 
The content of EGCG or PEGCG in each sample was calculated according to its standard curve, respectively. Thus, the conversion of EGCG can be obtained by the following equation:

$$
\% \text { Conversion }=\left(1-\text { content }_{\mathrm{EGCG}}\right) \times 100
$$

The product's yield was expressed as the weight of esters obtained/weight of esters $(\mathrm{g} / \mathrm{g})$ expected if EGCG is fully reacted.

\subsection{Evaluation of pH Stability in Alkalescent Condition}

Since the intestine system is alkalescent, it is necessary to assess the stability of EGCG and PEGCG under this condition. Ethanol/PBS $=80: 20(v / v, \mathrm{pH} 7.2)$ was used to offer the alkalescent condition, since PEGCG was insoluble in PBS.

Equivalent molar concentrations $(1.3 \mathrm{mM})$ of EGCG and PEGCG were incubated in Pyrex test tubes $(20 \times 1.6 \mathrm{~cm}$, i.d. $)$ in the open air at $37^{\circ} \mathrm{C}$, respectively, without any agitation. Aliquots $(20 \mu \mathrm{L})$ of the incubation solution was periodically $(1,2,5 \mathrm{~h})$ sampled and subjected to HPLC analysis, respectively. HPLC condition was the same as Item 2.5.

\subsection{Evaluation of Storage Stability}

To study the storage stability of EGCG and PEGCG powder, 1 molar of the two powders were kept in the open air at room temperature, respectively. After 60 days, aliquots of the two powders were sampled and subjected to HPLC analysis, respectively. The HPLC condition was the same as Item 2.5.

\subsection{Evaluation of Thermal Stability}

The thermal stability behaviors of EGCG and PEGCG were investigated on a TG apparatus (SDT Q600, TA Instruments, New Castle, DE, USA). About $3.0 \mathrm{mg}$ of each sample was used to perform the analysis by putting the powder on a TG pan. The sample was scanned from 30 to $400{ }^{\circ} \mathrm{C}$ with a heating rate of $10^{\circ} \mathrm{C} / \mathrm{min}$ under a nitrogen atmosphere.

\section{9. $\alpha$-Amylase Inhibition Assay}

The $\alpha$-amylase activity was measured using the method described by Nampoothiri with slight modifications [39]. For subsequent assays, equal volumes (100 $\mu \mathrm{L})$ of sample (EGCG and acarbose were dissolved in aqueous solution, and PEGCG in ethanol) and $100 \mu \mathrm{L}$ of porcine pancreatic $\alpha$-amylase $(0.5 \mathrm{mg} / \mathrm{mL})$ were incubated in microtubes at $25{ }^{\circ} \mathrm{C}$ for $10 \mathrm{~min}$. After pre-incubation, a volume of $1 \%$ starch solution in $10 \mathrm{mM}$ sodium phosphate buffer ( $\mathrm{pH}$ 7.2) was added to each tube and the samples were incubated at $25{ }^{\circ} \mathrm{C}$ for a further $10 \mathrm{~min}$. The reaction was stopped with $200 \mu \mathrm{L}$ of dinitrosalicyclic acid color reagent and tubes were incubated in boiling water for $5 \mathrm{~min}$. Once the samples were cooled to room temperature, $50 \mu \mathrm{L}$ was removed from each tube and transferred to the wells of 96-well microplate. The reaction mixture was diluted by adding $200 \mu \mathrm{L}$ of water to each well, and absorbance was measured at $540 \mathrm{~nm}$. Blank readings (no enzyme) were subtracted from each well, and results were compared to the control. The pharmacological inhibitor, acarbose, was included as a positive control. Two controls were used, which is, control 1 for EGCG and acarbose ( $20 \mu \mathrm{L}$ of PBS was added instead of the sample), and control 2 for PEGCG (20 $\mu \mathrm{L}$ of ethanol was added instead of the sample). The inhibition of $\alpha$-amylase was calculated as follows:

$$
\% \text { Inhibition }=\left(1-\frac{A_{\text {sample }}}{A_{\text {control }}}\right) \times 100
$$

\subsection{0. $\alpha$-Glucosidase Inhibition Assay}

The activity of $\alpha$-glucosidase was determined in a 96-well plate based on 4-Nitrophenyl beta-D-glucopyranoside (PNPG) as a substrate. A mixture of $100 \mu \mathrm{L}$ of enzyme solution $(1 \mathrm{U} / \mathrm{mL} \alpha$-glucosidase in $10 \mathrm{mM}$ sodium phosphate buffer) and $50 \mu \mathrm{L}$ of the specified 
concentration of samples (EGCG and acarbose dissolved in aqueous solution, and PEGCG in ethanol) was pre-incubated at $37^{\circ} \mathrm{C}$ for $10 \mathrm{~min}$; then $50 \mu \mathrm{L}$ of substrate solution $(5 \mathrm{mM}$ PNPG in $0.1 \mathrm{M}$ sodium phosphate buffer) was added to each well and incubated for $15 \mathrm{~min}$ at $37^{\circ} \mathrm{C}$ and stopped by adding $80 \mu \mathrm{L}$ of $0.2 \mathrm{M} \mathrm{Na}_{2} \mathrm{CO}_{3}$ [39]. The amount of PNP released was measured at $405 \mathrm{~nm}$. The controls (that do not include the enzyme) were prepared by adding PBS instead of the inhibitor solutions. Two controls were used, which were control 1 for EGCG and acarbose (20 $\mu \mathrm{L}$ of PBS was added instead of the sample), and control 2 for PEGCG (20 $\mu \mathrm{L}$ of ethanol was added instead of the sample). The inhibition of $\alpha$-glucosidase was calculated as follows:

$$
\% \text { Inhibition }=\left(1-\frac{A_{\text {sample }}}{A_{\text {control }}}\right) \times 100
$$

\subsection{Molecular Docking Simulation}

Molecular docking was used to study the binding details of the ligand-receptor complexes. AutoDock (version 4) [40,41] with Lamarckian genetic algorithm were used in the molecular docking experiments. The 3D crystal structures of $\alpha$-amylase (PDB ID: 1BLI) [42] and $\alpha$-glucosidase (PDB ID: 3A4A) (https://doi.org/10.1111/j.1742-4658.20 10.07810.x) were obtained from protein databank (RCSB PDB). The ligands were EGCG, PEGCG, and acarbose, and the 3D structure of the ligand was built by ChemBio3D Ultra 14.0. All possible binding models obtained from AutoDock were displayed to represent the binding conformation of drugs and enzyme. The binding models are clustered by binding energy from AutoDock with RMSD of $0.2 \mathrm{~nm}$. The contact region is defined as the protein within $0.5 \mathrm{~nm}$ of drugs. And the number of contact atom and amino acids are calculated. The contact surface area was calculated from the solution's accessible surface area. Binding conformations are displayed by VMD [43].

\section{Conclusions}

The results of this study suggest the possibility of a novel and efficient synthesis method of lipophilic EGCG palmitate. In this study, the usage of palmitoyl chloride, base, and solvent on the effect of the product yield and EGCG conversion were elucidated. Furthermore, the stability in alkalescent condition, storage stability, as well as the thermal stability of EGCG and PEGCG were analyzed and compared with each other. PEGCG was significantly more stable than EGCG, which contributed to its enhanced bioavailability and storage convenience. Moreover, the EGCG palmitate is capable of inhibiting $\alpha$-amylase and $\alpha$-glucosidase, indicating its potential as an antidiabetic prodrug. Furthermore, the oral toxicity tests of EGCG palmitate needs to be further studied.

Supplementary Materials: The following are available online in Figures S1-S7: 1H and 13C NMR spectra of PEGCG; Figure S3: HPLC-MS analysis of byproducts under the optimum condition; Figures S4 and S5: HPLC-MS analysis of product under the condition of 1 and 3 molar ratio of palmitoyl chloride, respectively; Figure S6: Identification of the oxidation product of EGCG under alkaline condition by HPLC-MS; Figure S7: Thermal gravity (TG) curves of (a) EGCG, and (b) PEGCG; Figure S7: Differential scanning calorimetry (DSC) analysis of PEGCG.

Author Contributions: Conceptualization, B.L. and W.Y.; investigation, B.L. and Z.K.; data curation, B.L. and Z.K.; writing-original draft preparation, B.L.; writing-review and editing, W.Y.; supervision, W.Y. All authors have read and agreed to the published version of the manuscript.

Funding: The authors appreciate funding from the National Key Research and Development Program of China (2017YFF0211000).

Institutional Review Board Statement: Not applicable.

Informed Consent Statement: Not applicable.

Data Availability Statement: The data presented in this study are available on request from the corresponding author. 
Conflicts of Interest: The authors declare no conflict of interest.

Sample Availability: Samples of the compounds are not available from the authors.

\section{References}

1. Wang, H.; Helliwell, K.; You, X. Isocratic elution system for the determination of catechins, caffeine and gallic acid in green tea using HPLC. Food Chem. 2000, 68, 115-121. [CrossRef]

2. Kurahashi, N.; Sasazuki, S.; Iwasaki, M.; Inoue, M.; Shoichiro, T.F.T.J. Green tea consumption and prostate cancer risk in Japanese men: A prospective study. Am. J. Epidemiol. 2008, 167, 71-77. [CrossRef] [PubMed]

3. Kuriyama, S.; Shimazu, T.; Ohmori, K.; Et, A. Green tea consumption and mortality due to cardiovascular disease, cancer, and all causes in japan: The ohsaki study. JAMA 2006, 296, 1255-1265. [CrossRef] [PubMed]

4. Raederstorff, D.G.; Schlachter, M.F.; Elste, V.; Weber, P. Effect of EGCG on lipid absorption and plasma lipid levels in rats. J. Nutr. Biochem. 2003, 14, 326-332. [CrossRef]

5. Takechi, R.; Alfonso, H.; Hiramatsu, N.; Ishisaka, A.; Tanaka, A.; Tan, L.B.; Lee, A.H. Elevated plasma and urinary concentrations of green tea catechins associated with improved plasma lipid profile in healthy Japanese women. Nutr. Res. 2016, 36, 220-226. [CrossRef]

6. Amarowicz, R.; Pegg, R.B.; Dykes, G.A.; Troszynska, A.; Shahidi, F. Antioxidant and antibacterial properties of extracts of green tea polyphenols. In Phenolic Compounds in Foods and Natural Health Products; Shahidi, F., Ho, C., Eds.; American Chemical Society: Washington, DC, USA, 2005; Volume 909, pp. 94-106.

7. Wolfram, S.; Raederstorff, D.; Preller, M.; Wang, Y.; Teixeira, S.R.; Riegger, C.; Weber, P. Epigallocatechin gallate supplementation alleviates diabetes in rodents. J. Nutr. 2006, 136, 2512-2518. [CrossRef]

8. Waltner-Law, M.E.; Wang, X.L.; Law, B.K.; Hall, R.K.; Nawano, M.; Granner, D.K. Epigallocatechin gallate, a constituent of green tea, represses hepatic glucose production. J. Biol. Chem. 2002, 277, 34933-34940. [CrossRef]

9. Kamiyama, O.; Sanae, F.; Ikeda, K.; Higashi, Y.; Minami, Y.; Asano, N.; Adachi, I.; Kato, A. In vitro inhibition of $\alpha$-glucosidases and glycogen phosphorylase by catechin gallates in green tea. Food Chem. 2010, 122, 1061-1066. [CrossRef]

10. Kale, A.; Gawande, S.; Kotwal, S.; Netke, S.; Roomi, W.; Ivanov, V.; Niedzwiecki, A.; Rath, M. Studies on the effects of oral administration of nutrient mixture, quercetin and red onions on the bioavailability of epigallocatechin gallate from green tea extract. Phytother. Res. 2010, 24, S48-S55.

11. Sang, S.; Lee, M.; Hou, Z.; Ho, C.; Yang, C.S. Stability of tea polyphenol (-)-epigallocatechin-3-gallate and formation of dimers and epimers under common experimental conditions. J. Agr. Food Chem. 2005, 53, 9478-9484. [CrossRef]

12. Hong, J.; Lu, H.; Meng, X.; Ryu, J.H.; Hara, Y.; Yang, C.S. Stability, cellular uptake, biotransformation, and efflux of tea polyphenol (-)-epigallocatechin-3-gallate in HT-29 human colon adenocarcinoma cells. Cancer Res. 2002, 62, 7241-7246. [PubMed]

13. Chow, H.S.; Hakim, I.A.; Vining, D.R.; Crowell, J.A.; Ranger-Moore, J.; Chew, W.M.; Celaya, C.A.; Rodney, S.R.; Hara, Y.; Alberts, D.S. Effects of dosing condition on the oral bioavailability of green tea catechins after single-dose administration of polyphenon $\mathrm{E}$ in healthy individuals. Clin. Cancer Res. 2005, 11, 4627. [CrossRef] [PubMed]

14. Nakagawa, K.; Miyazawa, T. Chemiluminescence-high-performance liquid chromatographic determination of tea catechin, (-)-epigallocatechin 3-gallate, at picomole levels in rat and human plasma. Anal. Biochem. 1997, 248, 41-49. [CrossRef] [PubMed]

15. Zhong, Y.; Shahidi, F. Lipophilized epigallocatechin gallate (EGCG) derivatives as novel antioxidants. J. Agr. Food Chem. 2011, 59, 6526-6533. [CrossRef] [PubMed]

16. Lam, W.H.; Kazi, A.; Kuhn, D.J.; Chow, L.M.C.; Chan, A.S.C.; Ping Dou, Q.; Chan, T.H. A potential prodrug for a green tea polyphenol proteasome inhibitor: Evaluation of the peracetate ester of (-)-epigallocatechin gallate [(-)-EGCG]. Bioorgan. Med. Chem. 2004, 12, 5587-5593. [CrossRef] [PubMed]

17. Liu, B.; Asadzadeh, B.; Yan, W. Solubility determination and modeling of EGCG peracetate in 12 pure solvents at temperatures from 278.15 to 318.15 K. J. Chem. Eng. Data 2019, 64, 5218-5224. [CrossRef]

18. Oliveira, D.A.; Adams, S.D.; Lee, L.H.; Murray, S.R.; Hsu, S.D.; Hammond, J.R.; Dickinson, D.; Chen, P.; Chu, T. Inhibition of herpes simplex virus type 1 with the modified green tea polyphenol palmitoyl-epigallocatechin gallate. Food Chem. Toxicol. 2013, 52, 207-215. [CrossRef]

19. Mori, S.; Miyake, S.; Kobe, T.; Nakaya, T.; Fuller, S.D.; Kato, N.; Kaihatsu, K. Enhanced anti-influenza A virus activity of (-)-epigallocatechin-3-O-gallate fatty acid monoester derivatives: Effect of alkyl chain length. Bioorg. Med. Chem. Lett. 2008, 18, 4249-4252. [CrossRef]

20. Matsumura, K.; Kaihatsu, K.; Mori, S.; Cho, H.H.; Kato, N.; Hyon, S.H. Enhanced antitumor activities of (-)-epigallocatechin-3O-gallate fatty acid monoester derivatives in vitro and in vivo. Biochem. Bioph. Res. Commun. 2008, 377, 1118-1122. [CrossRef]

21. Jung, J.H.; Yun, M.; Choo, E.; Kim, S.; Jeong, M.; Jung, D.; Lee, H.; Kim, E.; Kato, N.; Kim, B.; et al. A derivative of epigallocatechin3-gallate induces apoptosis via SHP-1-mediated suppression of BCR-ABL and STAT3 signalling in chronic myelogenous leukaemia. Br. J. Pharmacol. 2015, 172, 3565-3578. [CrossRef]

22. Matsumoto, Y.; Kaihatsu, K.; Nishino, K.; Ogawa, M.; Kato, N.; Yamaguchi, A. Antibacterial and antifungal activities of new acylated derivatives of epigallocatechin gallate. Front. Microbiol. 2012, 3, 53. [CrossRef] [PubMed]

23. Zhu, S.; Li, Y.; Ma, C.; Lou, Z.; Chen, S.; Dai, J.; Wang, H. Optimization of lipase-catalyzed synthesis of acetylated EGCG by response surface methodology. J. Mol. Catal. B Enzym. 2013, 97, 87-94. [CrossRef] 
24. Chen, P.; Tan, Y.; Sun, D.; Zheng, X. A novel long-chain acyl-derivative of epigallocatechin-3-O-gallate prepared and purified from green tea polyphenols. J. Zhejiang Univ.-Sci. A 2003, 4, 714-718. [CrossRef] [PubMed]

25. Baj, S.; Chrobok, A.; Gottwald, I. Application of solid-liquid phase transfer catalysis system for peroxyester synthesis: A kinetic study of hydroperoxides acylation in the presence of solid sodium carbonate. Appl. Catal. A Gen. 2002, 224, 89-95. [CrossRef]

26. Li, T.; Liu, J.; Zhang, X.; Ji, G. Antidiabetic activity of lipophilic (-)-epigallocatechin-3-gallate derivative under its role of $\alpha$-glucosidase inhibition. Biomed. Pharmacother. 2007, 61, 91-96. [CrossRef] [PubMed]

27. Tetko, I.V.; Bruneau, P. Application of ALOGPS to predict 1-octanol/water distribution coefficients, $\log$ P, and $\log \mathrm{D}$, of AstraZeneca in-house database. J. Pharm. Sci.-USA 2004, 93, 3103-3110. [CrossRef]

28. Krupkova, O.; Ferguson, S.J.; Wuertz-Kozak, K. Stability of (-)-epigallocatechin gallate and its activity in liquid formulations and delivery systems. J. Nutr. Biochem. 2016, 37, 1-12. [CrossRef]

29. Zhang, Y.; Chen, X.; Cheng, J.; Jin, C.; Zhang, Y. The reduction effect of dietary flavone C- and O-glycosides on the formation of acrylamide and its correlation and prediction with the antioxidant activity of Maillard reaction products. RSC Adv. 2014, 4, 24147-24155. [CrossRef]

30. Fu, Z.; Yoo, M.J.Y.; Zhou, W.; Zhang, L.; Chen, Y.; Lu, J. Effect of (-)-epigallocatechin gallate (EGCG) extracted from green tea in reducing the formation of acrylamide during the bread baking process. Food Chem. 2018, 242, 162-168. [CrossRef]

31. Etxeberria, U.; de la Garza, A.L.; Campión, J.; Martínez, J.A.; Milagro, F.I. Antidiabetic effects of natural plant extracts via inhibition of carbohydrate hydrolysis enzymes with emphasis on pancreatic alpha amylase. Expert Opin. Ther. 2012, 16, 269-297. [CrossRef]

32. Hsieh, S.; Shih, K.; Chou, C.; Chu, C. Evaluation of the efficacy and tolerability of miglitol in Chinese patients with type 2 diabetes mellitus inadequately controlled by diet and sulfonylureas. Acta Diabetol. 2011, 48, 71-77. [CrossRef] [PubMed]

33. Li, C.; Hung, Y.; Qamruddin, K.; Aziz, M.F.A.; Stein, H.; Schmidt, B. International noninterventional study of acarbose treatment in patients with type 2 diabetes mellitus. Diabetes Res. Clin. Pr. 2011, 92, 57-64. [CrossRef] [PubMed]

34. Bedekar, A.; Shah, K.; Koffas, M. Chapter 2-Natural products for type II diabetes treatment. In Advances in Applied Microbiology; Academic Press: San Diego, CA, USA, 2010; Volume 71, pp. 21-73.

35. Liu, B.; Yan, W. Lipophilization of EGCG and effects on antioxidant activities. Food Chem. 2019, 272, 663-669. [CrossRef] [PubMed]

36. Guo, Q.; Zhao, B.; Shen, S.; Hou, J.; Hu, J.; Xin, W. ESR study on the structure-antioxidant activity relationship of tea catechins and their epimers. Biochim. Biophys. Acta 1999, 1427, 13-23. [CrossRef]

37. Salah, N.; Miller, N.J.; Paganga, G.; Tijburg, L.; Bolwell, G.P.; Riceevans, C. Polyphenolic flavanols as scavengers of aqueous phase radicals and as chain-breaking antioxidants. Arch. Biochem. Biophys. 1995, 322, 339-346. [CrossRef]

38. Valcic, S.; Burr, J.A.; Timmermann, B.N.; Liebler, D.C. Antioxidant chemistry of green tea catechins. New oxidation products of (-)-epigallocatechin gallate and (-)-epigallocatechin from their reactions with peroxyl radicals. Chem. Res. Toxicol. 2000, 13, 801-810. [CrossRef]

39. Nampoothiri, S.V.; Prathapan, A.; Cherian, O.L.; Raghu, K.G.; Venugopalan, V.V.; Sundaresan, A. In vitro antioxidant and inhibitory potential of Terminalia bellerica and Emblica officinalis fruits against LDL oxidation and key enzymes linked to type 2 diabetes. Food Chem. Toxicol. 2011, 49, 125-131. [CrossRef]

40. Huey, R.; Morris, G.M.; Olson, A.J.; Goodsell, D.S. A semiempirical free energy force field with charge-based desolvation. J. Comput. Chem. 2007, 28, 1145-1152. [CrossRef]

41. Morris, G.M.; Huey, R.; Lindstrom, W.; Sanner, M.F.; Belew, R.K.; Goodsell, D.S.; Olson, A.J. AutoDock4 and AutoDockTools4: Automated docking with selective receptor flexibility. J. Comput. Chem. 2009, 30, 2785-2791. [CrossRef]

42. Machius, M.; Declerck, N.; Huber, R.; Wiegand, G. Activation of Bacillus licheniformis $\alpha$-amylase through a disorder $\rightarrow$ order transition of the substrate-binding site mediated by a calcium-sodium-calcium metal triad. Structure 1998, 6, 281-292. [CrossRef]

43. Humphrey, W.; Dalke, A.; Schulten, K. VMD: Visual molecular dynamics. J. Mol. Graph. 1996, 14, 33-38. [CrossRef] 